\title{
МОДЕЛЬ ПРОГНОЗА ВЕРОЯТНОСТИ РЕМИССИИ ДИФФУЗНОГО ТОКСИЧЕСКОГО ЗОБА
}

\author{
Дора С.В., Волкова А.Р., Дыгун О.Д., Абрамова И.М., Азизова К.В. \\ ФГБОУ ВО «ПСПбГМУ им. акад. И.П. Павлова», Санкт-Петербург
}

Раннее выявление группы риска рецидива ДТ3 крайне актуально, так как сохранение длительного тиреотоксикоза (ТT) приводит к развитию тиреотоксической кардиомиопатии и формированию жизнеугрожающих нарушений ритма (фибрилляции предсердий), сердечной недостаточности, остеопорозу, что ухудшает прогноз трудоспособности и продолжительности жизни больных. В настоящее время надежных критериев ремиссии ДТЗ нет. В связи с этим разработка и клиническая апробация методов ранней оценки вероятности ремиссии у больных ДТЗ - важная задача для оптимизации лечебной тактики.

ЦЕЛЬ: разработать критерий оценки вероятности ремиссии у больных ДТЗ.

МАТЕРИАЛЫ И МЕТОДЫ: бЫло проведено ретроспективное исследование, которое включало 515 пациентов с ДТ3 (404 (78,5\%) женщины и 111 (21,5\%) мужчин), диагностированным за период с 1970 до 2010 г. Средний возраст больных составил $41,96 \pm 0,60$ года $(41,15 \pm 0,66$ года у женщин, $45,25 \pm 0,60$ года у мужчин; $\mathrm{p}=0,01)$. Все они получали терапию тиреостатиками на протяжении 12-18 месяцев. После отмены лечения пациенты наблюдались каждые 6 месяцев в течение 5 лет. Для разработки математической модели оценки вероятности ремиссии ДТЗ на фоне стандартной антитиреоидной терапии проведен пошаговый регрессионный анализ, на основании которого построена дискриминантная функция $D$, позволившая классифицировать больных на две группы. Группа 1 - значение D менее 3,26, группа 2 - значение D равно 3,26 и более.

PЕзУЛЬТАТЫ: выделены шесть значимых дискриминантных переменных, которые влияют на прогноз ремиссии ДТЗ: исходный объем щитовидной железы $\left(\mathrm{cm}^{3}\right)$, наличие офтальмопатии, возраст начала заболевания (годы), соотношение уровней св. Т3 и св. Т4, исходные уровни антител к рецептору тиреотропного гормона, табакокурение. На основании отбора наиболее информативных признаков создано уравнение дискриминантной функции $\mathrm{D}$. Если значение $\mathrm{D}<3,26$, то пациент будет иметь высокую вероятность ремиссии, если значение $D \geq 3,26$, то вероятность ремиссии низкая. В проспективном исследовании показано, что расчетная модель оценки вероятности ремиссии ДТЗ обладает высокой чувствительностью (86\%) и специфичностью (84\%).

ВЫВОДЫ: разработанная модель оценки ремиссии ДТЗ позволяет прогнозировать течение заболевания в момент постановки диагноза и оптимизировать лечебную тактику.

КЛЮЧЕВЫЕ СЛОВА: диффузный токсический зоб; тиреотоксикоз; ремиссия диффузного токсического зоба; модель оценки вероятности ремиссии. 